\title{
Upper Bounds on Rare K and B Decays from MFV
}

\section{Thorsten Ewerth ${ }^{* \dagger}$}

Institute of Theoretical Physics, Sidlerstrasse 5, CH-3012 Bern, Switzerland

E-mail: tewerth@itp.unibe.ch

Recently, we studied the branching ratios of rare $K$ and $B$ decays in models with Minimal Flavor Violation (MFV) using the presently available information from the universal unitarity triangle analysis and from the measurements of $\operatorname{Br}\left(B \rightarrow X_{s} \gamma\right), \operatorname{Br}\left(B \rightarrow X_{s} l^{+} l^{-}\right)$and $\operatorname{Br}\left(K^{+} \rightarrow \pi^{+} v \bar{v}\right)$ [1]. We analyzed in detail possible scenarios with positive or negative interference of Standard Model (SM) and New Physics contributions. In particular, we derived upper bounds on various rare decays and pointed out an interesting triple correlation between $B \rightarrow X_{s} \gamma, B \rightarrow X_{s} l^{+} l^{-}$and $K^{+} \rightarrow \pi^{+} v \bar{v}$ present in MFV models.

International Europhysics Conference on High Energy Physics

July 21st - 27th 2005

Lisboa, Portugal

* Speaker.

${ }^{\dagger}$ Based on collaboration with C. Bobeth, M. Bona, A. J. Buras, M. Pierini, L. Silvestrini and A. Weiler [1]. 
The MFV models considered here are defined as in [2], which means that (i) flavor and CP violation is entirely governed by the CKM matrix, and (ii) the relevant operators entering the effective Lagrangians of weak decays are those already present in the $\mathrm{SM}^{1}$. Under these assumptions any weak decay amplitude can be written as follows [4],

$$
A_{\text {decay }}=\sum_{i} B_{i} \eta_{\mathrm{QCD}}^{i} V_{\mathrm{CKM}}^{i} F_{i}(v), \quad F_{i}(v)=F_{\mathrm{SM}}^{i}+\Delta F_{i}(v)
$$

where the master functions $F_{i}(v)$, with $v$ denoting collectively the parameters of a given MFV model, contain besides the heavy degrees of freedom of the SM also the New Physics contributions via the real functions $\Delta F_{i}(v)$. Furthermore, $B_{i}$ parameterize hadronic matrix elements of local operators, and $\eta_{\mathrm{QCD}}^{i}$ describe the QCD evolution of the functions $F_{i}(v)$ from the high- to the low-energy scale. Both are universal in MFV and known from SM calculations. For the CKM parameters $V_{\text {CKM }}^{i}$ we use the results of the universal unitary triangle analysis of the UIfit collaboration [5]. In the following table we collect the most important rare decays relevant for our numerical analysis together with the master functions $F_{i}(v)$ characterizing them,

$$
\begin{array}{ll}
K^{+} \rightarrow \pi^{+} v \bar{v}, K_{L} \rightarrow \pi^{0} v \bar{v}, B \rightarrow X_{d, s} v \bar{v} & X(v)=C(v)+B^{v \bar{v}}(v) \\
K_{\mathrm{L}} \rightarrow \mu^{+} \mu^{-}, B_{d, s} \rightarrow l^{+} l^{-} & Y(v)=C(v)+B^{l \bar{l}}(v) \\
B \rightarrow X_{s} \gamma & D^{\prime}(v), E^{\prime}(v) \\
B \rightarrow X_{s} l^{+} l^{-} & Y(v), Z(v)=C(v)+\frac{1}{4} D(v), E(v), D^{\prime}(v), E^{\prime}(v)
\end{array}
$$

Here, the master functions $E(v), D(v)$ and $B(v)$ can be set to a very good approximation equal to their SM values ${ }^{2}$, and the functions $D^{\prime}(v)$ and $E^{\prime}(v)$ can be traded for $C_{7}^{\text {eff }}(v)$ being the relevant quantity at the low-energy scale for both $B \rightarrow X_{s} \gamma$ and $B \rightarrow X_{s} l^{+} l^{-}$. Therefore the only free parameters for the considered decays in the MVF models are the functions $C_{7}^{\text {eff }}(v)$ and $C(v)$. The strategy for our numerical analysis is then $(i)$ to constrain the functions $C_{7}^{\text {eff }}(v)$ and $C(v)$ through the measured branching ratios of $B \rightarrow X_{s} \gamma, B \rightarrow X_{s} l^{+} l^{-}$and $K^{+} \rightarrow \pi^{+} v \bar{v}$, and (ii) to compute expectation values and upper bounds following from the constraints on these two parameters for the other decays of the above given table.
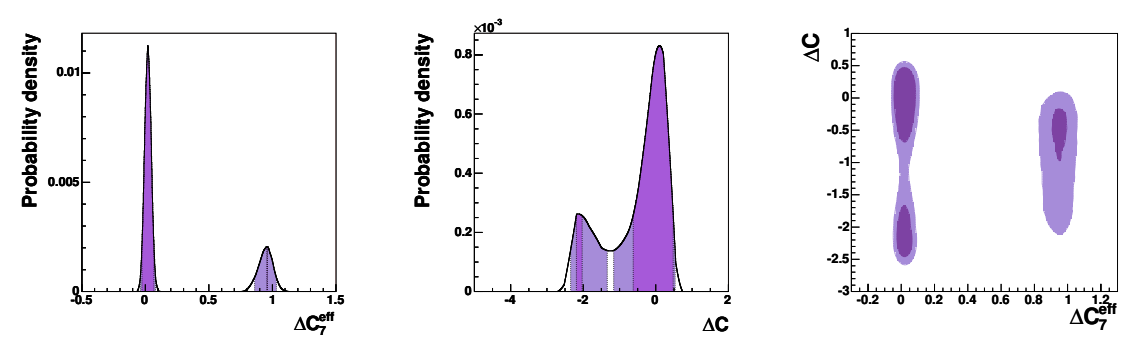

Figure 1: Probability density functions (pdfs) for $\Delta C_{7}^{\mathrm{eff}}, \Delta C$ and $\Delta C_{7}^{\mathrm{eff}} v \mathrm{vs} \Delta$. Dark (light) areas correspond to the $68 \%$ (95\%) probability regions. The $S M$ predictions are $C_{7}^{\text {eff }} \approx-0.33$ and $C \approx 0.81$.

\footnotetext{
${ }^{1}$ This is a special case of the effective field theoretical approach given in [3].

${ }^{2}$ In fact, varying $\Delta D(v)$ in the range $\pm D_{\mathrm{SM}}$ has only little impact on our numerical results. Furthermore, we checked that as long as the functions $\Delta B^{l \bar{l}}(v)$ and $\Delta B^{v \bar{v}}(v)$ are of comparable size as the SM ones our results do not change sizably. Finally, $\Delta E(v)$ can be neglected due to a tiny pre-factor accompanying it in the analytic expressions for the branching ratios.
} 

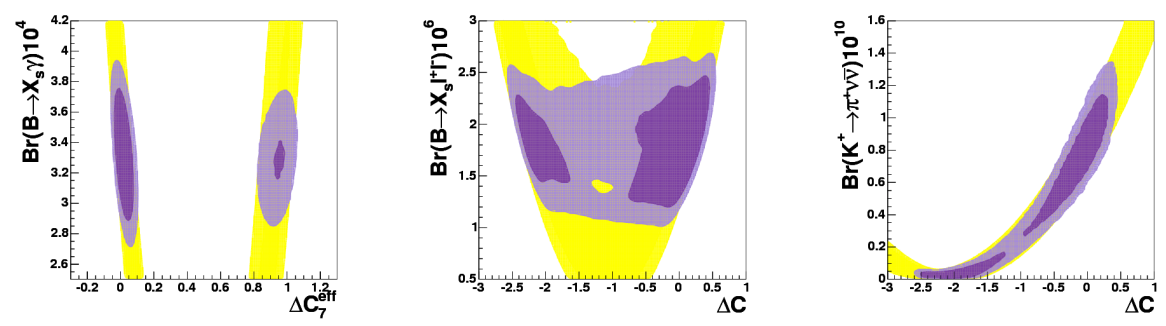

Figure 2: Pdfs for the branching ratios used to constrain $C_{7}^{\text {eff }}$ and $C$ as functions of these parameters. The $S M$ predictions are $\operatorname{Br}\left(B \rightarrow X_{s} \gamma\right)_{E_{\gamma}>1.8 \mathrm{GeV}} \approx 3.5 \times 10^{-4}, \operatorname{Br}\left(B \rightarrow X_{s} l^{+} l^{-}\right)_{1<q^{2}(\mathrm{GeV})<6} \approx 1.6 \times 10^{-6}$ and $\operatorname{Br}\left(K^{+} \rightarrow \pi^{+} v \bar{v}\right) \approx 8.3 \times 10^{-10}$. Dark (light) areas correspond to the $68 \%$ (95\%) probability regions, and very light ones to the range without using the experimental information.

Figure 1 shows that there exist SM-like solutions with $C_{7}^{\text {eff }}(v)<0$ and $C(v)>0$. But we also find another solution for $C_{7}^{\text {eff }}(v)$ which corresponds to reversing the sign of the SM prediction. This opposite sign solution is however disfavored because $\operatorname{Br}\left(B \rightarrow X_{s} l^{+} l^{-}\right)$tends to become larger than the experimental value [6]. Furthermore, we find a second solution for $C(v)$ which also corresponds to reversing the sign of its SM prediction. The suppression of this opposite sign solution follows from the experimental data on $\operatorname{Br}\left(K^{+} \rightarrow \pi^{+} v \bar{v}\right)$. The third plot shows the correlation between $\Delta C_{7}^{\text {eff }}(v)$ and $\Delta C(v)$. There are two solutions for $\Delta C(v)$ for the SM-like solution of $\Delta C_{7}^{\text {eff }}(v)$ but only one solution for $\Delta C(v)$ for the opposite sign solution of $C_{7}^{\text {eff }}(v)$. The first plot in Figure 2 gives the pdf for $\operatorname{Br}\left(B \rightarrow X_{s} \gamma\right)$ vs $\Delta C_{7}^{\text {eff }}(v)$ with the running charm quark mass used in the low-energy matrix elements and a photon cut-off $E_{\gamma}=1.8 \mathrm{GeV}$. Again we see that the opposite sign solution is disfavored. The second plot shows the pdf for $\operatorname{Br}\left(B \rightarrow X_{s} l^{+} l^{-}\right)$vs $\Delta C(v)$ in the low- $q^{2}$ region in order to avoid the theoretical uncertainties due to $c \bar{c}$ resonances, and the last one $\operatorname{Br}\left(K^{+} \rightarrow \pi^{+} v \bar{v}\right)$ vs $\Delta C(v)$. As can be seen, the signs of $C(v)$ and $C_{7}^{\text {eff }}(v)$ cannot be determined at present, but with the ongoing reduction of the experimental and theoretical uncertainties of rare $B$ decays it will become possible to eliminate the opposite sign solution of $C_{7}^{\text {eff }}(v)$. However, it will be difficult to determine the sign of $C(v)$ from $\operatorname{Br}\left(B \rightarrow X_{s} l^{+} l^{-}\right)$alone. This ambiguity can then be resolved by a more precise measurement of $\operatorname{Br}\left(K^{+} \rightarrow \pi^{+} v \bar{v}\right)$. We also note that eliminating the opposite sign solution
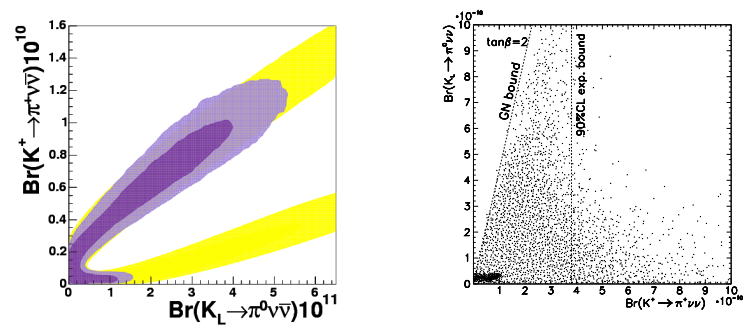

Figure 3: Left: Pdf for $\operatorname{Br}\left(K^{+} \rightarrow \pi^{+} v \bar{v}\right)$ vs $\operatorname{Br}\left(K_{L} \rightarrow \pi^{0} v \bar{v}\right)$ within MFV models. Dark (light) areas correspond to the $68 \%$ (95\%) probability regions, and very light ones to the range without using the experimental information. Right: Scatterplot for $\operatorname{Br}\left(K^{+} \rightarrow \pi^{+} v \bar{v}\right)$ vs $\operatorname{Br}\left(K_{L} \rightarrow \pi^{0} v \bar{v}\right)$ within the general MSSM. 

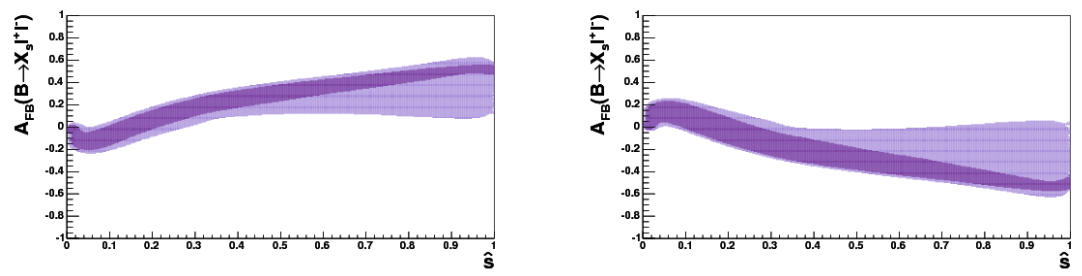

Figure 4: Forward-backward asymmetry of $B \rightarrow X_{s} l^{+} l^{-}$for the $S M$-like solution for $C_{7}^{\text {eff }}$ with $\Delta C>-1$ (left) and $\Delta C<-1$ (right). Dark (light) areas correspond to the $68 \%$ (95\%) probability regions.

of $C(v)$ by means of this decay would basically also eliminate the opposite sign solution of $C_{7}^{\text {eff }}(v)$. Figure 3 shows $\operatorname{Br}\left(K^{+} \rightarrow \pi^{+} v \bar{v}\right)$ vs $\operatorname{Br}\left(K_{L} \rightarrow \pi^{0} v \bar{v}\right)$. Within the MFV models (left plot) one has a very strong correlation and the neutral decay mode is always one order of magnitude smaller than the charged one. The right plot illustrates that this is completely different in non-MFV models. It has been obtained by taking the full flavor structure of the squark sector of the general MSSM into account [7]. Here, the neutral decay mode can easily be of the same order as the charged one. Other interesting sensitive probes of $C_{7}^{\text {eff }}(v)$ and $C(v)$ are the forward-backward asymmetries of inclusive and exclusive semileptonic $B$ decays. Figure 4 shows the shape of the asymmetry for $B \rightarrow X_{s} l^{+} l^{-}$depending on the sign of $C(v)$ for the SM-like solution of $C_{7}^{\text {eff }}(v)$. A measurement of this observable would clearly help in determining the sign of $C(v)$. The table given below displays our upper bounds for rare decays in MFV at $95 \%$ probability together with the corresponding SM predictions and the available experimental information.

\begin{tabular}{|c|c|c|c|c|}
\hline Branching Ratios & MFV (95\%) & SM (95\%) & SM (68\%) & exp. \\
\hline $\operatorname{Br}\left(K^{+} \rightarrow \pi^{+} v \bar{v}\right) \times 10^{11}$ & $<11.9$ & $<10.9$ & $8.3 \pm 1.2$ & $\left(14.7_{-8.9}^{+13.0}\right)$ \\
\hline $\operatorname{Br}\left(K_{\mathrm{L}} \rightarrow \pi^{0} v \bar{v}\right) \times 10^{11}$ & $<4.6$ & $<4.3$ & $3.1 \pm 0.6$ & $<2.86 \times 10^{4}$ \\
\hline $\operatorname{Br}\left(K_{\mathrm{L}} \rightarrow \mu^{+} \mu^{-}\right) \mathrm{SD} \times 10^{9}$ & $<1.4$ & $<1.2$ & $0.9 \pm 0.1$ & - \\
\hline $\operatorname{Br}\left(B \rightarrow X_{s} v \bar{v}\right) \times 10^{5}$ & $<5.2$ & $<4.1$ & $3.7 \pm 0.2$ & $<64$ \\
\hline $\operatorname{Br}\left(B \rightarrow X_{d} v \bar{v}\right) \times 10^{6}$ & $<2.2$ & $<1.9$ & $1.5 \pm 0.2$ & - \\
\hline $\operatorname{Br}\left(B_{s} \rightarrow \mu^{+} \mu^{-}\right) \times 10^{9}$ & $<7.4$ & $<5.9$ & $3.7 \pm 1.0$ & $<2.7 \times 10^{2}$ \\
\hline $\operatorname{Br}\left(B_{d} \rightarrow \mu^{+} \mu^{-}\right) \times 10^{10}$ & $<2.2$ & $<1.8$ & $1.0 \pm 0.3$ & $<1.5 \times 10^{3}$ \\
\hline
\end{tabular}

We conclude that the present constraints from $B \rightarrow X_{s} \gamma, B \rightarrow X_{S} l^{+} l^{-}$and $K^{+} \rightarrow \pi^{+} v \bar{v}$ do not allow for large enhancements of the branching ratios of other rare $K$ and $B$ decays with respect to the SM predictions. Any violation of these upper bounds signals new sources of flavor and $\mathrm{CP}$ violation and/or the presence of operators beyond those relevant in the SM.

\section{References}

[1] C. Bobeth et al., Nucl. Phys. B 726 (2005) 252.

[2] A. J. Buras, P. Gambino, M. Gorbahn, S. Jäger and L. Silvestrini, Phys. Lett. B 500 (2001) 161

[3] G. D’Ambrosio, G. F. Giudice, G. Isidori and A. Strumia, Nucl. Phys. B 645 (2002) 155.

[4] A. J. Buras, Acta Phys. Polon. B 34 (2003) 5615

[5] M. Bona et al. [UTfit Collaboration], hep-ph/0509219.

[6] P. Gambino, U. Haisch and M. Misiak, Phys. Rev. Lett. 94 (2005) 061803.

[7] A. J. Buras, T. Ewerth, S. Jäger and J. Rosiek, Nucl. Phys. B 714 (2005) 103. 